\title{
Microstructure of stomaflex based magnetic elastomers
}

\author{
(C) M. Balasoiu ${ }^{* * * *}$, M.L. Craus ${ }^{* *, * * *}$, E.M. Anitas ${ }^{* * * * * * *}$, \\ I. Bica ${ }^{* * * *}$, J. Plestil ${ }^{* * * * *}$, A.I. Kuklin ${ }^{* * *}$ \\ * National Institute of Physics and Nuclear Engineering, \\ Bucharest, Romania \\ ** National Institute of Research and Development for Technical Physics, \\ lasi, Romania \\ *** Joint Institute of Nuclear Research, \\ Dubna, Moscow region, Russia \\ **** West University of Timisoara, Department of Electricity and Magnetism, \\ Timisoara, Romania \\ ***** Institute of Macromolecular Chemistry, Academy of Sciences of the Czech Republic, \\ Prague, Czech Republic \\ E-mail: balasoiumaria@yahoo.com
}

\begin{abstract}
Stomaflex elastomers filled with two types of magnetic particles (nano- and micro-sized) were investigated. It was observed that doping with $\mathrm{Fe}_{3} \mathrm{O}_{4}$ nanoparticales and applying a magnetic field during the polymerisation process led to a significant change in the local structure of the elastomer. Decreases in the quasi-crystalline phase concentration, in the average size of the crystalline blocks, and in the ordering distance were observed after doping the elastomer with magnetite nanoparticles. After filling the polymer with $\mathrm{Fe}_{3} \mathrm{O}_{4}$ nanoparticles, yet the elastomer fractal dimension changes. For the elastomer filled with a large amount of Fe microparticles ( $75 \%$ particle concentration) a texture effect is observed, and this effect is larger for the samples polymerised in a magnetic field. At all microparticle concentrations, these elastomers exhibit surface fractal structure.
\end{abstract}

\section{Introduction}

Recent interest in rubber-like active-material devices has stimulated increased effort in both basic and applied research on new elastomer technology. Magnetic elastomers belong to a specitic class of so-called smart materials, because they can respond to changes in their environment. They are composed of magnetic particles and a low-permeability matrix (Fig. 1). Application of the external magnetic field may form a structure inside the material, or a structure already present in the material may change.

Finely divided filler particles have been used for many decades as reinforcing agents in elastomers, and the resulting macroscopic elastic properties have traditionally proven useful in tires, seals, and passive damping devices.

The combination of magnetic and elastic properties leads to various phenomena which can be initiated by the external magnetic field [1-7]. These phenomena have opened up new possibilities for technological applications, such as: 1) magnetoelastic composites; with particles made of magnetostrictive hard or soft ferromagnetic material; 2) magnetorheological elastomers, for applications in the aerospace and automotive industries as actuators on antifriction components; 3) heat-shrinkable elastic ferromagnets with variable magnetic and conductive properties.

Other applications that utilise magnetic polymer nanocomposites are currently emerging at a high rate. Examples include magnetic actuation in microelectromechanical systems and medical devices, thermal actuation through electromagnetic power harvesting, and magnetically actuated morphing structures.

The magneto-elastic properties of composites are not merely a sum of elasticity of the polymer and stiffness and magnetic properties of the filler, but also the result of a complex synergy of several effects, relevant at different length scales and detectable by different techniques.

Many studies of the observed reinforcing effect of magnetic fillers have approached the problem from a magnetomechanical point of view, and investigated the microscopic properties through study of the magneto-elastic responses of the composity [1-4]. Less well understood, however, are the effects of the interactions between the filler and the surrounding polymer on submicroscopic length scales. Such length scales are ideally suited for small angle scattering investigations [8-10].

In this paper, variations in the magnetic elastomer's microscopic properties due to filling the polymer matrix with: 1) magnetite nanoparticles and 2) Fe microparticles, are investigated by means of $X$-ray diffraction (XRD), small angle neutron scattering (SANS) and small angle $X$-ray scattering (SAXS) methods.

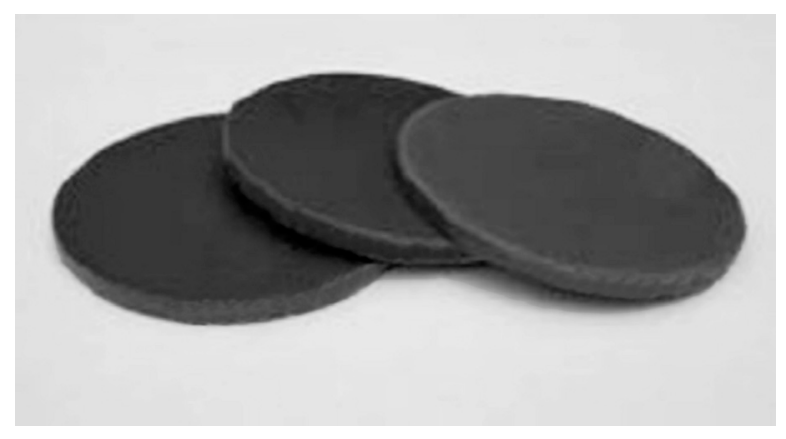

Fig. 1. Example of magnetic elastomer samples. 


\section{Experimental}

Two types of samples were prepared at the Department of Electricity and Magnetism, West University of Timisoara [11-13]. One type of sample was composed of an oil based, 2.2\% particle volume concentration $\mathrm{Fe}_{3} \mathrm{O}_{4}$ ferrofluid with oleic acid as a surfactant (Fig. 2) [14,15], embedded in a polymer matrix formed from dimethylsiloxane and dibutyltindilaurate bensyl silicate, and polymerised in either zero field (sample $A$ ) or in an applied magnetic field of $B=135.9 \mathrm{mT}$ (sample $B$ ). The second type consisted of the same polymer matrix with embedded Fe microparticles, obtained by thermal decomposition of $\mathrm{Fe}_{2}(\mathrm{CO})_{9}$ (Fig. 3). In this case, the polymerisation process was performed in zero field and in an applied magnetic field $B=156.5 \mathrm{mT}$.

From electron micorscopy, images of the $\mathrm{Fe}_{3} \mathrm{O}_{4}$ ferrofluid (Fig. 2) and of the $\mathrm{Fe}$ particles obtained by thermal decomposition of $\mathrm{Fe}_{2}(\mathrm{CO})_{9}$ (Fig. 3) were obtained. Size-distribution histograms yielded a mean di-

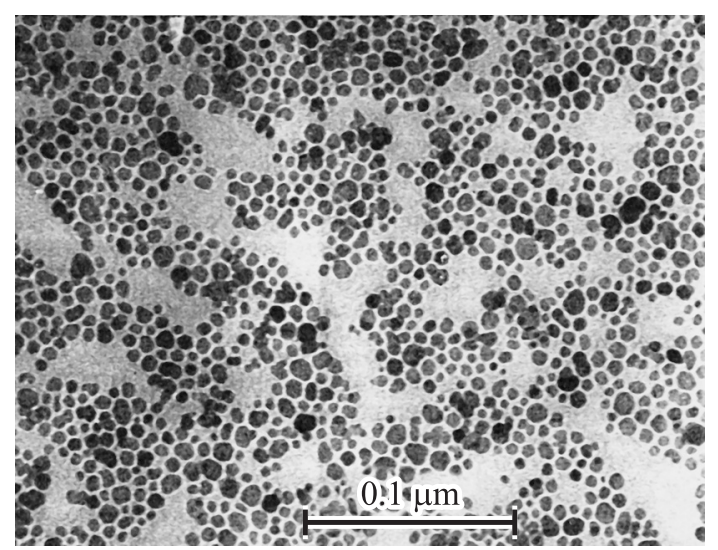

Fig. 2. Electron microscopy image $\mathrm{Fe}_{3} \mathrm{O}_{4}$ particles in ferrofluid sample.

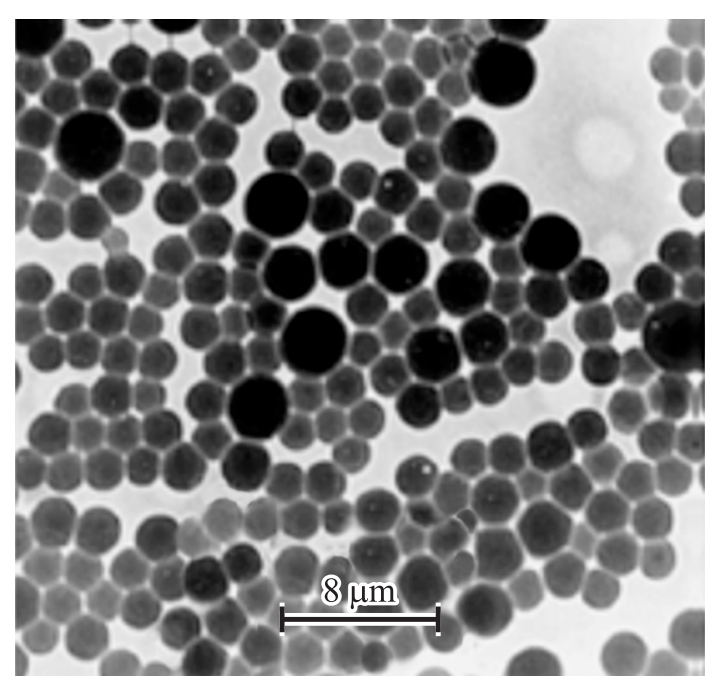

Fig. 3. Electron microscopy image of iron microparticles in mineral oil [16].

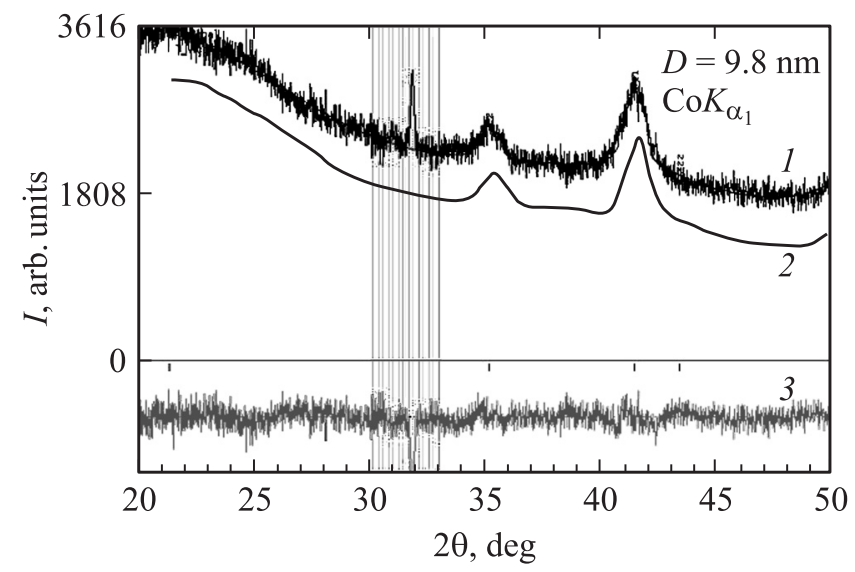

Fig. 4. Observed (1) and calculated (2) diffractograms (upperside); difference between observed and calculated diffractograms (3) for $\mathrm{Fe}_{3} \mathrm{O}_{4}$ particles.

ameter of $\langle D\rangle=11.4 \mathrm{~nm}$ with a standard deviation of $\sigma=1.94 \mathrm{~nm}$ (Fig. 2), and a mean of $\langle D\rangle=2.24 \mu \mathrm{m}$ with a standard deviation of $0.33 \mu \mathrm{m}$ (Fig. 3).

In order to determine the phase composition, lattice micro-distortions, and the average coherent length (average size of mosaic blocks), the samples were investigated by XRD using DRON diffractometers. The magnetite samples were studied with $\mathrm{Co}_{\alpha}$-radiation, and the Fe-doped samples with $\mathrm{MoK}_{\alpha}$-radiation. XRD data were handled using Ceck Cell and Rietveld software.

The sample were also studied using SAXS and SANS scattering methods. SAXS experiments were performed with a Rigaku spectrometer using a pinhole camera (Molecular Metrology SAXS System) attached to a microfocused $X$-ray beam generator (Osmic MicroMax 002). The camera was equipped with a multiwire, gas-filled area detector with on active area diameter of $20 \mathrm{~cm}$. Two experimental setups were used to cover the $q$ range of $0.007-1.1 \AA^{-1}$.

The SANS experiments were carried out on the YUMO diffractometer [17] at the IBR-2 pulsed reactor (JINR, Dubna) in the $Q$-range of about $0.005-0.3 \AA^{-1}$.

\section{Results}

3.1. Magnetic elastomers with $\mathrm{Fe}_{3} \mathrm{O}_{4}$ nanoparticles. For the elastomers doped with $\mathrm{Fe}_{3} \mathrm{O}_{4}$ particles of about $10 \mathrm{~nm}$ in average size, the XRD data revealed that the elastomers contained basically the same concentration of $\mathrm{Fe}_{3} \mathrm{O}_{4}$, but the average sizes of coherent blocks and microstrains were different. It was also established that both samples contain a small amount of foreign phase (Fig. 4).

Data from the SAXS measurements indicated strong difference between the structures of doped and undoped samples. We observed the systematic appearance of a Bragg diffraction peak in the SAXS plots, in the region of large $q$ values (near $q=0.9 \AA^{-1}$, see in Fig. 5). 


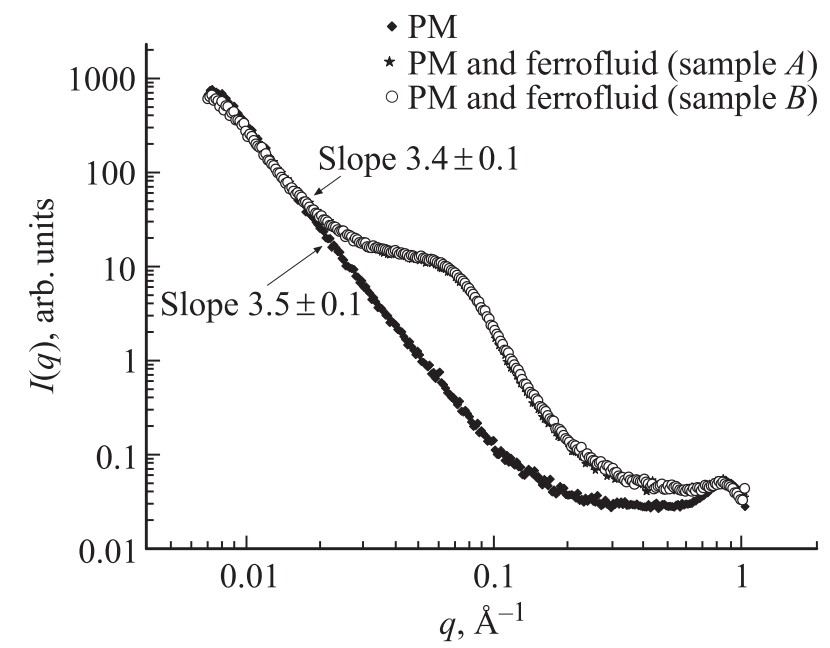

Fig. 5. SAXS experimental curves from samples $A, B$ and a simple elastomer, obtained at Rigaku spectrometer at IMC (Prague) [9].

Doping with $\mathrm{Fe}_{2} \mathrm{O}_{4}$ particles leads to a significant change in the local structure of an elastomer, causing a decrease in the quasi-crystalline phase concentration (Fig. 3) and the average size of the crystalline blocks (Table 1). Fitting the observed maximum corresponding to $q \approx 0.9 \AA^{-1}$ with a pseudo-Voigt profile appears to show that the elastomer is fragmented into small particles. The average size of elastomer blocks and the ordering distance decreased when magnetite nanoparticles were introduced in the elastomer, and increased when a magnetic field was applied during the polymerisation process (Table 1).

Table 1. Variation of the centre of gravity position $\theta$, the average size of crystalline blocks $\Delta$, and the ordering distance $D$ in the elastomer matrix

\begin{tabular}{l|c|c|c}
\hline \multicolumn{1}{c|}{ Sample } & $\theta$ & $\Delta, \mathrm{nm}$ & $D, \mathrm{~nm}$ \\
\hline Elastomer matrix & $11.56 \pm 0.02$ & $2.73 \pm 0.04$ & $0.763 \pm 0.002$ \\
Elastomer with & $11.71 \pm 0.01$ & $2.38 \pm 0.13$ & $0.756 \pm 0.001$ \\
$\mathrm{Fe}_{3} \mathrm{O}_{4}$ particles (po- \\
lymerization without \\
magnetic field) \\
$\begin{array}{l}\text { Elastomer with } \\
\mathrm{Fe}_{3} \mathrm{O}_{4} \text { particles } \\
\text { (polymerization } \\
\text { with magnetic field) }\end{array}$ & $11.41 \pm 0.05$ & $2.73 \pm 0.14$ & $0.775 \pm 0.007$ \\
\end{tabular}

The maxima observed at $q \approx 0.08 \AA^{-1}$ (Fig. 5) corresponds to the presence of $100 \AA$ nanoparticles, in agreement with the results of Klokkenburg et al. [18].

The SANS scattering intensities of a stomaflex créme polymer matrix reveal a power-law behaviour $I(q) \approx q^{-\alpha}$ with the exponent $\alpha<4$. In the present case, when $\alpha<4$, the fractal dimension is given by the formula $D_{s}=6-\alpha$ [19]. Consequently, the polymer matrix exhibited the behaviour of a surface fractal object with fractal dimension $D_{s}=2.5 \pm 0.1$. The magnetic elastomers created upon introduction of the ferrofluid (sample $A$ ) and sample $B$ created in applied magnetic field were fractal objects with a surface fractal dimension $D_{s}=2.7 \pm 0.1$ (Fig. 6).

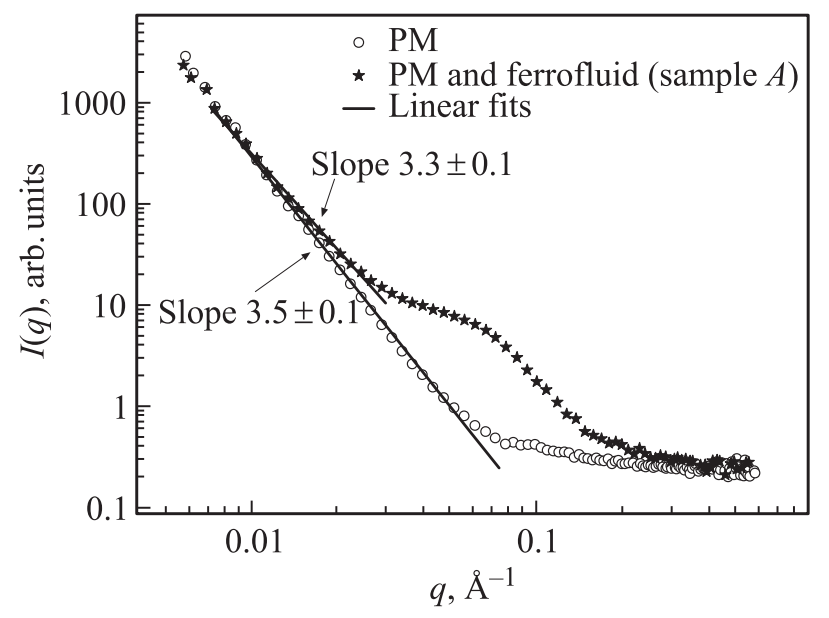

Fig. 6. Scattering intensity experimental curves and linear fits for polymer matrix (PM) and polymer matrix with embedded $\mathrm{Fe}_{3} \mathrm{O}_{4}$ ferrofluid $(\mathrm{PM}+$ ferrofluid $)$.

In the case of the SAXS experimental curves, the following results were obtained: 1) surface fractal dimension of $D_{s}=2.5 \pm 0.1$ for the polymer matrix; 2) surface fractal dimension of $D_{s}=2.6 \pm 0.1$ for the elastomer with $\mathrm{Fe}_{3} \mathrm{O}_{4}$ ferrofluid; 3) surface fractal dimension of $D_{s}=2.6 \pm 0.1$ the elastomer with $\mathrm{Fe}_{3} \mathrm{O}_{4}$ ferrofluid polymerised in an external magnetic field.

3.2. Magnetic elastomers with Fe microparticles. XRD diffractograms of elastomers with Fe microparticles (in various concentrations) give the relative intensities of different lattice planes. These diffractograms were taken for samples composed of different concentrations of $\mathrm{Fe}$, and polymerised in the presence or absence of

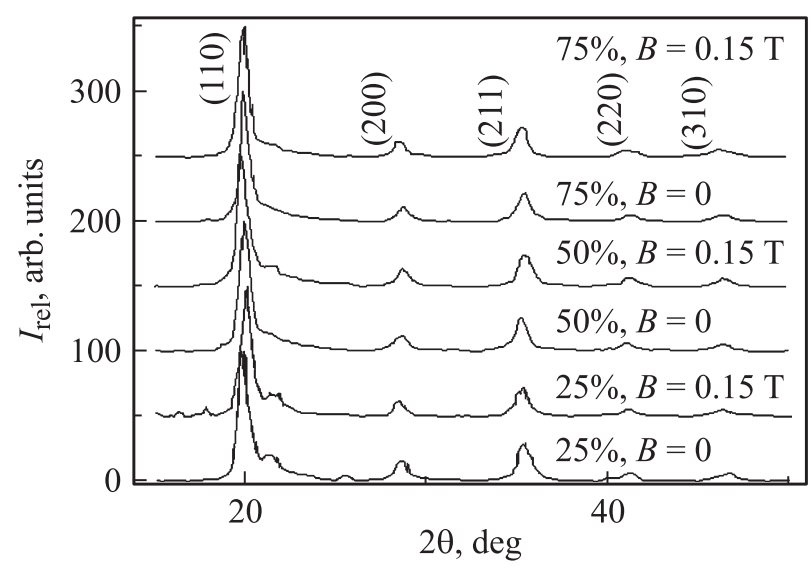

Fig. 7. Diffractograms of elastomer samples with Fe microparticles (in various concentrations, polymerised in and without magnetic field), using $\mathrm{Mo}_{\alpha}$ radiation. 
Table 2. Modification of the relative intensities of different lattice planes with the variation of $\mathrm{Fe}$ microparticle concentration and polymerisation in magnetic field

\begin{tabular}{c|c|c|c|c|c}
\hline \multirow{2}{*}{$\begin{array}{c}\text { Fe } \\
\text { (concentration, \%) }\end{array}$} & \multicolumn{5}{c}{ Relative intensities $\left(\mathrm{Mo} K_{\alpha}\right)$} \\
\cline { 2 - 6 } & $(110)$ & $(200)$ & $(211)$ & $(220)$ & $(310)$ \\
\hline \multicolumn{5}{c}{$B=0$} \\
25 & 100 & 14.4 & 26.4 & 5.8 & 9.9 \\
50 & 100 & 12.5 & 21.6 & 5.0 & 7.9 \\
75 & 100 & 13.7 & 28.3 & 8.9 & 8.3 \\
\multicolumn{5}{c}{ B } \\
50 & 100 & $9.15 \mathrm{~T}$ & 22.5 & 4.4 & 5.5 \\
75 & 100 & 11.6 & 24.8 & 7.3 & 5.5 \\
& 100 & 8.7 & 18.1 & 6.0 & 12.2 \\
Fe standard & 100 & 15 & 26.5 & 3.9 & 8.7
\end{tabular}

a magnetic field (Fig. 7). The principal maxima were attributed to $\mathrm{Fe}$ (b.c.c.). Weak maxima near (110) of the b.c.c. phase can be attributed to a f.c.c. austenite type structure.

In Table 2, the change in the relative intensities of different lattice when changing Fe microparticle concentration and polymerisation magnetic field is presented.

The relative intensities of the lattice planes (200), (211), (220), and (310) decreased with increasing Fe concentration for the samples synthesised without a magnetic field, until the $\mathrm{Fe}$ concentration reached $50 \%$. At $75 \% \mathrm{Fe}$, the relative intensities of (200), (211), and (220) still decreased, but the relative intensity of the (310) maxima increased. This result shows that a small crystallographic texture effect can take place for higher Fe concentration in the sample. For the samples polymerised in an external magnetic field, a general decrease of relative intensities of the (200) and (211) maxima was observed, indicating that the texture effect

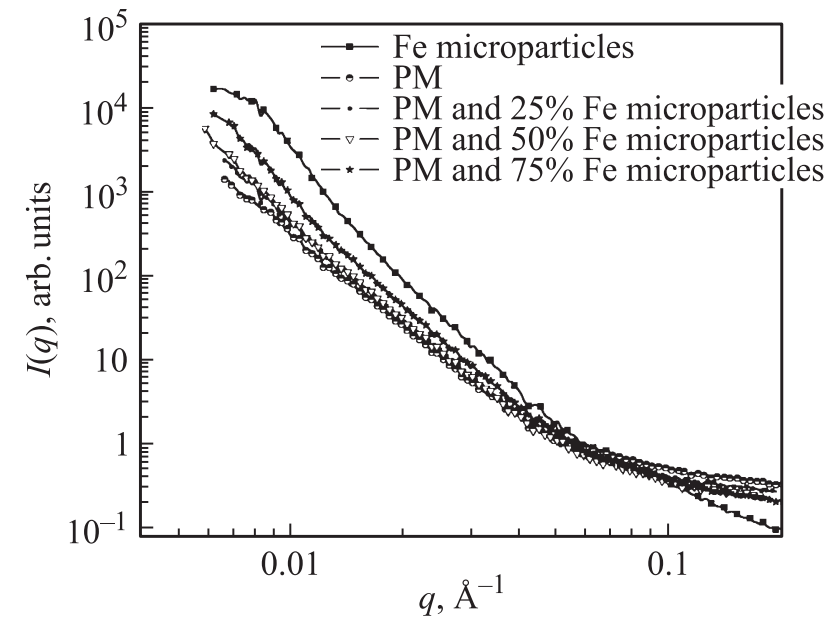

Fig. 8. SANS experimental curves of elastomer with $\mathrm{Fe}$ microparticles, stomaflex elastomer matrix and Fe particles. is larger due to more uniform relative orientation of the Fe particles. That means, the magnetic field enhances the degree of orientation in the samples, as the (110) plane becomes perpendicular to the magnetic field.

The SANS scattering intensities of a stomaflex créme polymer matrix with $\mathrm{Fe}$ microparticles also exhibit powerlaw behaviour $I(q) \approx q^{-\alpha}$ with the exponent $3<\alpha<4$ (Fig. 8). For elastomer samples with $25 \% \mathrm{Fe}$ particle concentration, a surface fractal dimension of $D_{s}=2.3 \pm 0.1$ was obtained; at $50 \% \quad D_{s}=2.2 \pm 0.1 ;$ and at $75 \%$ $D_{s}=2.1 \pm 0.1$.

Power-law behaviour with an exponent of $\alpha=4$ was observed for the scattering intensity of $\mathrm{Fe}$ particles, showing the particles to be smooth. In this case, using the Porod law $I=\frac{2 \pi \rho^{2}}{Q^{4}} S$, where $S$ is the mean specific surface of the particles, a mean radius of $\langle R\rangle=2.00 \pm 0.25 \mu \mathrm{m}$ is obtained for the Fe particles, in very good agreement with scanning electron microscopy data.

\section{Conclusions}

Stomaflex elastomers filled with two types of magnetic particles (nano- and micro-sized) were investigated.

It was established that doping with $\mathrm{Fe}_{3} \mathrm{O}_{4}$ nanoparticles and application of a magnetic field during the polymerisation process cause a significant change of the local structure of the elastomer. Decreases in the quasi-crystalline phase concentration, in the average size of the crystalline blocks, and in the ordering distance are present in the elastomer doped with magnetite nanoparticles, while by the application of a magnetic field during the polymerisation process, increases in the above mentioned parameters are detected.

The stomaflex polymer matrix is charactreised as a surface fractal with a dimension of about $D_{s}=2.5 \pm 0.1$. After filling the polymer with $\mathrm{Fe}_{3} \mathrm{O}_{4}$ nanoparticles, the magnetic elastomer fractal dimension changes. With the application of a magnetic field during polymerisation, the fractal dimension remains constant.

For the elastomer filled with a large amount of $\mathrm{Fe}$ microparticles (75\% particle concentration), a texture effect is detected, and the effect is larger for samples polymerised in a magnetic field. At all microparticle concentrations, the elastomer is characterised as a surface fractal.

We acknowledge Dr. M. Lita for XRD measurements and Dr. A.Kh. Islamov for helpful discussions and remarks. The grants of Romanian Plenipotentiary in JINR are acknowledged.

\section{References}

[1] Yu.L. Raikher, O.V. Stolbov. Pis'ma Zh. Tekhn. Fiz. 26, 4, 47 (2000).

[2] L. Lanotte, G. Ausanio, C. Hison, V. Ianotti, C. Luponio, C. Luponio, Jr. J. Opt. Adv. Mater. 6, 2, 523 (2004). 
[3] S. Abramchuk, E. Kramarenko, G. Stepanov, L.V. Nikitin, G. Filipcsei, A.R. Khokhlov, M. Zrínyi. Polymers Adv. Tech. 18, 11, 883 (2007).

[4] G.V. Stepanov, D.Yu. Borin, Yu.L. Raikher, P.V. Melenev, N.S. Perov. J. Phys.: Cond. Matter 20, 204121 (2008).

[5] S. Ahmed. J. Mater. Sci. 25, 4933 (1990).

[6] A. Emmerling, P. Wang, G. Popp, A. Beck, J. Frike. J. Phys. 13, 357 (1993).

[7] I. Bica, H.J. Choi. Int. J. Mod. Phys. B 22, 29, 5042 (2008).

[8] A. Botti, W. Pyckhout-Hintzen, V. Urban, J. Kohlbrecher, D. Richter, E. Straube. Appl. Phys. A 74 (Suppl), S 513 (2002).

[9] M. Balasoiu, M.L. Craus, A.I. Kuklin, J. Plestil, V. Haramus, A.H. Islamov, R. Erhan, E.M. Anitas, M. Lozovan, V. Tripadus, C. Petrescu, D. Savu, I. Bica. J. Opt. Adv. Mater. 10, 11, 2932 (2008).

[10] M. Balasoiu, E.M. Anitas, I. Bica, V.A. Osipov, O.L. Orelovich, D. Savu, S. Savu, R. Erhan, A.I. Kuklin. Opt. Adv. Mater.: Rapid Commun. 2, 11, 730 (2008).

[11] I. Bica. J. Ind. Eng. Chem. 12, 5, 806 (2006).

[12] I. Bica. J. Magn. Magn. Mater. 241, 196 (2002).

[13] I. Bica. J. Ind. Eng. Chem. 11, 2, 299 (2007).

[14] D. Bica. RO Patent 90078 (1985).

[15] M. Balasoiu, B. Grabcev, D. Bica. Rom. Rep. Phys. 47, 3-5, 319 (1995).

[16] I. Bica. Mater. Sci. Eng. B 98, 89 (2003).

[17] A.I. Kuklin, A.Kh. Islamov, V.I. Gordely. Neutron News 16, 3, 16 (2005).

[18] M. Klokkenburg, B.H. Erné, A. Wiedenmann, A.V. Petukhov, A. Philipse. Phys. Rev. E 75, 051408 (2007).

[19] H.D. Bale, P.W. Schmidt. Phys. Rev. Lett. 53, 6, 596 (1984). 